\title{
Allergic Cutaneous Angiitis
}

National Cancer Institute

\section{Source}

National Cancer Institute. Allergic Cutaneous Angiitis. NCI Thesaurus. Code C35119.

Inflammation of the small vessels of the skin that is mediated by the immune system. 